Clinical Decision Support for Visual Diagnosis

Arthur Papier, MD; Tener Goodwin Veenema, $P b D, M P H$, $M S, C P N P$

Logical lmages, Inc., Rochester, New York USA

During the United States anthrax attacks of 2001, patients' lives depended upon the astuteness of the clinician. The danger highlighted the need to enhance clinical detection and diagnostic accuracy of clinicians in case of a biological attack. Victims of a biological or chemical attack present to the healthcare system with a constellation of symptoms that often includes visual evidence of the exposure. The ability to differentiate between benign and life-threatening skin manifestations is critical. While skin disease is a frequent complaint affecting a large percentage of people, most physicians and nurses receive limited training in dermatology, and frequently, are not confident in their ability to diagnose and manage dermatological diseases. Diagnoses of rare diseases and their manifestations produced by bioterrorism that have non-specific early findings create even more challenges.

A computer-based Clinical Decision Support System (CDSS) refers to computer programs designed to assist clinicians in decision-making. VisualDx (Logical Images, Inc., Rochester, New York) is a CDSS designed to provide diagnostic support and referencing capabilities for the image-rich areas of medicine, including general dermatology, infectious diseases, drug reactions, and the results of bioterrorism. VisualDx allows searching the database using patient findings, cutaneous morphology, and rash distribution. Designed for emergency and public health clinicians, the system merges the world's largest collection of digitized images with textual information to guide diagnosis and treatment, including conditions caused by the use of weapons of mass destruction. VisualDx facilitates the early detection of diseases by providing instant access to the information needed to rapidly diagnose and begin treatment.

Keywords: bioterrorism; chemicals; cutaneous manifestations; database; diagnosis; exposure; images; VisualDx; weapons of mass destruction

Prebosp Disast Med 2005:20(2):s93

\section{Long-term Environmental and Health Effects of Weapons with Depleted Uranium \\ G. Tiroler \\ Sweden}

Weapons containing so-called depleted uranium (DU) were used first in the Gulf war in 1991, then in the Balkans, Afghanistan, and currently in Iraq. Simultaneously, there have been reports of unexpected increases in the incidence of malignancies and birth defects among veterans, their children, and civilians in the areas where these weapons have been deployed. While a score of other explanations have been suggested, like dust, pollution, vaccinations, posttraumatic stress disorder, and others, the one recurring and common factor for all those theaters of war has been DU.

DU is both chemically and radiologically toxic. It is a heavy metal with clear nephrotoxic properties, but it also emits alpha rays. One single atom of uranium ${ }_{238}$ inside the body can damage the adjacent cells and eventually cause cancer. The international states using DU arms certainly are not interested in having them banned by contravening international conventions. They question the evidence produced on the dangers inherent in DU for its long-term environmental and health effects including damaging the genetic code for generations to come. Not only have investigations into the biological and clinical consequences of DU been hindered, but the presentations of findings concerning such weapons also have been hindered as well.

This review summarizes the current knowledge gathered from field observations, follow-up studies, and laboratory experiments, including the testimonies at an international, scientific conference in Stockholm in April 2004. This presentation argues the case for deeper clinical and in-vitro studies, as well as for the immediate cessation of the use of DU weapons. Keywords: depleted uranium (DU); environmental effects; health effects; weapons

Prebosp Disast Med 2005;20(2):s93

Late Respiratory Complications of Sulfur Mustard Poisoning in Iranian Veterans

\section{Hefazi, ${ }^{1}$ D. Attaran, ${ }^{2}$ M. Mabmoudi, ${ }^{3}$}

M. Balali-Mood ${ }^{4}$

1. Department of Respiratory Medicine, GHaem Hospital, School of Medicine, Mashhad, Iran

2. Iran Immunology Research Center, Bu-ali Research Institute

3. Iran Medical Toxicology Center, Imam Reza Hospital, School of Medicine, Mashhad, Iran

4. Janbezen (Veteran's) Organization, Iran

Introduction: Mustard gas, or sulfur mustard (SM), is an alkylating chemical warfare agent that was widely used during World War I and in the Iran-Iraq conflict. Delayed respiratory complications are the greatest cause of longterm disability among SM casualties.

Objective: To study late toxic effects of SM on the respiratory system of severely affected Iranian veterans.

Methods: Respiratory examination, spirometry, arterial blood gas (ABG) analysis, and high-resolution computed tomography (HRCT) of the chest were performed on all severely SM-poisoned veterans in the province of Khorasan, Iran. HRCT abnormalities were classified into four grades based on the number of lung lobes involved. $A B G$ values were compared with spirometric results, as well as with the severity grades of HRCT abnormalities, using Spearman's rank correlation test.

Results: Forty male patients (mean age $43.8 \pm 9.8$ years) with confirmed SM poisoning 16-20 years previously were studied. Main respiratory complications were diagnosed as chronic obstructive pulmonary disease (35\%), bronchiectasis $(32.5 \%)$, asthma (25\%), large airway narrowing $(15 \%)$, pulmonary fibrosis $(7.5 \%)$, and simple chronic bronchitis $(5 \%)$. The overall pattern of spirometry was obstructive in $57.5 \%$, restrictive in $22.5 \%$, mixed in $15 \%$, and normal in $5 \%$ of the patients. Mild, moderate, and severe hypoxemia was found in $67.5 \%, 27.5 \%$, and $0 \%$ of the patients, respectively. While there was a significant correlation $(p<0.05)$ between $A B G$ values and spirometric results, the severity grades of HRCT abnormalities revealed a significant correlation $(p<0.05)$ only with $\mathrm{PaO} 2$ value. 
Conclusion: $\mathrm{SM}$ is an incapacitating chemical warfare agent with several devastating long-term effects on human health. SM-induced respiratory complications tend to progress over the years. While spirometry is a valuable diagnostic tool for evaluation of pulmonary impairment during regular follow-ups, $\mathrm{ABG}$ and HRCT are more objective and should be considered for evaluation of the severity and for diagnosis of the respiratory complications. Keywords: complications; effects; evaluation; follow up; respiratory; sulfur mustard (SM)

Prebosp Disast Med 2005;20(2):s93-s94

\section{A Modification of the JUMPStart Triage Algorithm Used for a Large American City A. Cooper; ${ }^{1}$ G. Foltin; ${ }^{2}$ M. Tunik; ${ }^{2}$ B. Kaufman; ${ }^{3}$ G. Asaeda; ${ }^{3}$ D. Gonzalez;, J. Clair ${ }^{3}$ \\ 1. Columbia University/Harlem Hospital, New York, New York USA \\ 2. New York University/Bellevue Hospital, New York, New York USA \\ 3. Fire Department, City of New York, New York, New York USA}

The JUMPStart Triage Algorithm (JTA) for children substitutes bag-valve-mask ventilation (BVM) for airway repositioning as used in the Simple Triage and Rapid Treatment Algorithm (START) paradigm for adults. However, the BVM will not be feasible in a hot zone or even in the triage and staging area (TSA) of a casualty-collection point (CCP), if resuscitation equipment is not readily available.

A modification of the JTA was developed for children $<5$ years of age that may be necessary operationally under field conditions when a BVM is not readily available. The following principles guided development of this pediatric triage algorithm: (1) it must embrace all hazards; (2) it must be part of the existing triage process for the general population; (3) it must be able to be incorporated easily and quickly into the existing municipal disaster triage process; and (4) the JTA cannot be used as it currently exists, due to the lack of resuscitation equipment in the hot zone, but the START paradigm must remain the foundation for pediatric triage.

Based on these principles, the following approach has been developed for pediatric triage. All pediatric patients must be transferred from the hot zone to a decontamination area prior to definitive triage at a TSA within the CCP. Only dead or moribund patients will remain in the hot zone. Pediatric patients able to walk are tagged as "Green". Pediatric patients unable to walk are initially tagged as "Red" if breathing occurs spontaneously or upon airway repositioning, but are initially tagged as "Black" if breathing is absent. Patients initially tagged as "Red" remain so if their respiratory rate remains $<20$ or $>40$ cycles per minute (bpm), but are definitively tagged as "Yellow" if their respiratory rate is $>20$ and $<40 \mathrm{cpm}$, pulse is palpable, and movement is present and purposeful. Children definitively tagged as "Red" or "Yellow", then, receive expedited off-site transport. However, definitive "Black" tagging and forensic transport cannot occur until a child initially tagged as "Black" has failed to respond to two rescue breaths via BVM administered as soon as possible after transfer to the TSA.
Conclusion: It is speculated that this paradigm will be effective in saving children's lives under field conditions in mass-casualty events.

Keywords: children; JUMPStart Triage Algorithm; mass-casualty event; triage

Prebosp Disast Med 2005;20(2):s94

\section{Mass Toxicological Incidents (MTIs)-Are Local Procedures Necessary? \\ P. Gula; ${ }^{1}$ J. Nitecki; ${ }^{1}$ W. Hladki; ${ }^{2}$ L. Brongel; ${ }^{2}$ \\ B. Kozanecka-Muzyk ${ }^{3}$ \\ 1. State Fire, Krakow, Poland \\ 2. Emergency Medicine and Multiple Trauma Department, Medical College Jagiellonian University, Poland \\ 3. Rydygiera Hospital, Krakow, Poland}

With a population of $>1$ million, Krakow is a major town in Poland. The Krakow area has a high risk for chemical events related to the chemical industry and hazardous material transport. During the last two years, there were three major chemical incidents, of which the largest involved 37 patients. Based on international experience, local emergency procedures were developed. These are based on the cooperation of numerous institutions, including State Fire and Rescue Service, Rescue Coordination Center, Regional Toxicological Information Center, Krakow's emergency medical services, and hospital emergency departments.

Procedures include detection, on-site rescue procedures including decontamination if needed, transportation, and hospital treatment. The important part of the system is cautious training, including drills and the evaluations of this training. The authors will present algorithms for masstoxicological incidents and the way they adapted to local response system, and their follow-up after implementing the plan in drills and chemical incidents.

Keywords: chemical; emergency; planning; Poland; preparedness; procedures

Prebosp Disast Med 2005;20(2):s94

Assessment of Psychosocial Impacts of a Chemical Weapons Attack on Civilian Population of Sardasht, Iran

S. Khateri; $M$. Soroush ${ }^{2}$

1. Janbazan Medical and Engineering Research Center (JMERC), Janbazan (Veterans) Organization, Director, Chemical Warfare (CW) Victims Research Unit, Iran

2. Janbazan Medical and Engineering Research Center (JMERC), Iran

Introduction: Individuals sustaining wartime injuries that survive the experience nevertheless may develop serious long-term health problems as a result. Previous studies of this phenomenon, which focused primarily on the effects of trauma due to conventional armament, have documented various clinically defined categories of chronic disorders in battle-injured personnel. However, a similar body of medical literature has not been developed as extensively for persons affected by nuclear, biological or chemical (NBC) weapons. In particular, there have been few in-depth analyses of long-term psychosocial effects among civilian victims of NBC attacks. However, the increasing probability of 